\title{
Corela
}

Cognition, représentation, langage

18-1 | 2020

Vol. $18, \mathrm{n}^{\circ} 1$

\section{Les associations évoquées par les mots : collecte, analyse, exploitation}

\section{Michèle Debrenne}

\section{(2) OpenEdition}

1 Journals

\section{Édition électronique}

URL : http://journals.openedition.org/corela/11426

DOI : 10.4000/corela. 11426

ISSN : 1638-573X

Éditeur

Cercle linguistique du Centre et de l'Ouest - CerLICO

Référence électronique

Michèle Debrenne, «Les associations évoquées par les mots : collecte, analyse, exploitation », Corela [En ligne], 18-1 | 2020, mis en ligne le 26 juin 2020, consulté le 29 juin 2020. URL : http:// journals.openedition.org/corela/11426; DOI : https://doi.org/10.4000/corela.11426

Ce document a été généré automatiquement le 29 juin 2020.

\section{(c) (i) (2)(2)}

Corela - cognition, représentation, langage est mis à disposition selon les termes de la licence Creative Commons Attribution - Pas d'Utilisation Commerciale - Partage dans les Mêmes Conditions 4.0 International. 


\title{
Les associations évoquées par les mots : collecte, analyse, exploitation ${ }^{1}$
}

\author{
Michèle Debrenne
}

\section{Introduction}

1 Allant à l'encontre de l'opinion généralement admise chez les linguistes français, Claude Hagège affirmait qu'une "langue constitue aussi une manière de penser, une façon de voir le monde, une culture" (Hagège 2012). Approuvant ou niant l'existence d'une "vision du monde" reflétée par les mots, depuis Humboldt, Sapir et Whorf, les linguistes sont partagés sur la nature des relations entre la langue et la pensée. Ce qui semble évident au linguiste naï (la "saudade" portugaise ne correspond pas plus à "nostalgie" que le "spleen" anglais) ne l'est plus aux yeux du chercheur féru d'universalisme.

2 Sans approfondir ici cette polémique, nous présenterons une méthode d'objectivation de relations lexicales le plus souvent ignorées des lexicographes, qui reflètent, dans une certaine mesure, le lien entre le lexique et l'image du monde. Il existe différentes méthodes d'étude de ces connotations partagées par les locuteurs d'une langue, que les dictionnaires classiques ne retiennent pas toujours: citons ici l'analyse des points de vue sous-tendus par les mots (Raccah, 2014), l'introspection linguistique des bilingues (voir les travaux de J.-M. Dewaele, F. Grosjean et A. Pavlenko), et l'ethnolexicologie, basée, elle aussi, sur le contraste des langues en contact, qui étudie des mots-clés, en vue de découvrir si derrière eux se cachent des valeurs culturelles caractéristiques de la langue-culture où ils ont été observés, en invoquant des faits linguistiques aussi bien que non linguistiques (Peeters, 2013).

3 On peut également, et c'est notre approche, appliquer la méthode psycholinguistique de fixation de la première réponse à un stimulus lexical et créer ainsi ce que l'on appelle des "dictionnaires d'associations évoquées par les mots" (cf. par ex. Nelson et 
al, 1998). A l'heure actuelle il existe un certain nombre de ces dictionnaires, ils seront présentés en détail plus bas.

\section{Les dictionnaires d'associations évoquées par les mots : quelques repères historiques}

4 Les premiers à utiliser des expériences d'associations dans l'étude du discours ont été les chercheurs allemands A. Thumb et K. Marbe (1901). A l'origine, ce type d'expérience était surtout utilisé pour étudier les processus psychiques, notamment ceux de généralisation ou de reproduction. Dans une seconde période, ces expériences ont été utilisées en linguistique, pour étudier la sémantique et délimiter ce qui y était dû aux facteurs psychiques et ce qui était lié à la langue.

5 On distingue plusieurs types d'expériences d'associations : les associations libres, dans lesquelles la forme de la réaction demandée (substantif, verbe etc., éventuellement idiome ou même, ces derniers temps, émoticône) n'est pas indiquée, et les associations dirigées, quand on doit donner une réponse d'un type précis (par ex. uniquement des adjectifs). Par ailleurs les associations peuvent être uniques (une seule réaction par stimulus) ou en chaîne, quand on demande aux participants de donner plusieurs (en général de trois à cinq) associations au stimulus donné. Dans ce cas interviennent des relations plus complexes, car chaque association suivante est motivée non seulement par le stimulus mais également par la réaction qui vient d'y être donnée, ou à leur combinaison. Dans le cas des dictionnaires d'associations évoquées par les mots, on demande de fournir une réaction libre par stimulus.

6 Les chercheurs apportent un soin particulier à l'établissement de la liste de stimuli. En règle générale, on propose 100 mots à chaque participant à l'expérience, choisis parmi les mots pleins les plus fréquents de la langue pour laquelle on se propose de créer ce dictionnaire. Dans les expériences de grande envergure, on propose à chaque personne une liste aléatoire de 100 mots établie d'après une sélection plus grande, de 1000 mots par exemple. Concernant le nombre de participants, même si, ainsi qu'il a été prouvé (УФимцевА et al. 2016), des résultats significatifs sont obtenus à partir de 200 participants, on préfère étendre l'expérience de manière à obtenir au moins 500 réponses par stimulus.

7 Le premier dictionnaire d'associations verbales a été publié en 1910 (Kent, Rosanoff 1910). La liste de stimuli utilisés comprenait 100 mots hautement fréquents et 1000 personnes ont participé à l'expérience. Par la suite, d'autres études ont été entreprises aux États-Unis avec la même liste de stimuli, par exemple W. A. Russel et J. J. Jenkins (1954). Un ouvrage collectif dirigé par L. Postman réunit tous les travaux connus à son époque sur les normes associatives de l'anglais, du français et de l'allemand (1970).

8 Il existe un type particulier de dictionnaires d'associations, qu'il est convenu d'appeler "thesaurus" et qui présente le plus grand intérêt. A l'heure actuelle, on en connaît pour l'anglais et le russe. Le thesaurus anglais a été créé par une équipe de psychologues dirigée par J. Kiss à Édimbourg (Kiss 1970); son but était d'étudier la structure du lexique interne des locuteurs de l'anglais. Ce type de dictionnaire diffère des autres en ce qu'il est établi en trois étapes, chacune à partir d'une liste de stimuli différents : les mille premiers mots sont choisis par les chercheurs, la deuxième et la troisième liste découlent des réactions obtenues à l'étape précédente. On forme non seulement un 
dictionnaire direct, comportant les stimuli et toutes les réactions qu'ils ont provoquées, mais également un dictionnaire inverse, dans lequel les liens associatifs sont présentés dans l'autre sens: les vedettes des articles du dictionnaire sont les réactions, pour chacune d'elles sont indiqués, dans un ordre décroissant, tous les stimuli qui les ont provoquées.

Le "Dictionnaire associatif russe", publié en six (КАРАУЛОВ et al. 1994-98) puis deux tomes (2002) s'est inspiré du projet des chercheurs d'Édimbourg (triple expérience, dictionnaire direct et inverse) : c'est donc un thesaurus. Sa popularité en Russie a donné naissance à une nouvelle branche de la linguistique, que l'on peut nommer "linguistique des associations". On y trouve d'une part de très nombreuses études, ponctuelles, longitudinales ou transversales, des normes associatives dans telle ou telle communauté linguistique, par exemple les travaux de E. Guts sur les associations évoquées par les mots chez les adolescents de la ville sibérienne d'Omsk (Гуц 2004) ou l'étude des normes associatives des écoliers de Saratov (гольдин 2009), grand projet longitudinal commencé en 1998. D'autre part les chercheurs utilisent cet outil comme instrument commode de mise en évidence et d'analyse de la langue-culture dans ses manifestations verbales chez les locuteurs ordinaires, de l'image linguistique du monde et son caractère systémique, de ses modifications au cours de la vie de la société, de la construction d'un modèle du lexique en réseau d'associations. Plusieurs bases de données associatives ont été créées et sont désormais accessibles sur internet, comme, par exemple, le "Dictionnaire associatif des langues slaves", qui porte sur les langues bulgare, biélorusse, russe et ukrainienne (УФимцЕВА et al. 2004), ou celui des normes associatives de l'espagnol (Puig 2001).

Enfin, en 2008 les chercheurs de l'Institut de Linguistique de Moscou ont lancé une nouvelle série d'expériences psycholinguistiques, dans le but de créer un thesaurus du russe du XXI ${ }^{e}$ siècle qui permettra d'évaluer l'évolution de la conscience linguistique des locuteurs de cette langue à la lumière des immenses changements qui ont affecté le pays depuis les années 1990, date à laquelle les données du premier dictionnaire avaient été récoltées. Dans ce cadre, on dispose d'ores et déjà de trois bases de données, l'une pour la partie européenne du pays, la deuxième pour la partie centrale, et la dernière pour la Sibérie et l'extrême Orient russe. La liste des 1000 stimuli est la même pour les trois zones, ces bases de données sont entièrement informatisées et les nouveaux dictionnaires ont commencé à paraitre (РУсскиЙ... 2014 t1 et t2, ШАПОшниковА 2014, 2015). L'équipe du professeur Sternin, à Voronège, propose une approche synthétique des résultats obtenus grâce aux diverses enquêtes psycholinguistiques qu'ils présentent comme des "dictionnaires psycholinguistiques » (РУдАКОвА et al. 2018).

\section{Les dictionnaires français d'associations évoquées par les mots}

11 Pour le français, on dispose à l'heure actuelle des dictionnaires suivants (accessibles en ligne sur le site www.dictaverf.nsu.ru) : 


\subsection{Le Dictionnaire des associations verbales du français (DAF)}

Ce dictionnaire a été réalisé entre 2007 et 2010 par une équipe mixte (Université de Novossibirsk - Paris-3). Une liste de stimuli de 1000 mots a été établie, sur la base de la liste de fréquence prise sur http://eduscol.education.fr. Seuls les substantifs, adjectifs, verbes et adverbes pleins ont été retenus. Comme il était prévu que l'étude des normes associatives du français s'étende par la suite à différentes zones de la Francophonie, et notamment à l'Afrique francophone, une liste supplémentaire de 100 lexèmes a été ajoutée, qui aurait dû permettre de comparer les champs associatifs des Français hexagonaux et de leurs contemporains en Afrique. En réalité cette étude n'a pas été réalisée et les mots ainsi introduits ont joué un rôle de "bruit" pour certains locuteurs hexagonaux qui ne les connaissaient pas. L'exigence méthodologique d'obtenir des réponses provenant du plus grand nombre de villes françaises, et l'éloignement géographique de l'auteur du dictionnaire ont conditionné le choix d'Internet, par l'intermédiaire d'un site dédié, comme méthode de collecte de données, à la différence des expériences psycholinguistiques traditionnelles qui s'opéraient sur papier avec entrée ultérieure des données sur ordinateur. Une étude a par ailleurs montré que la méthode employée pour la collecte des données dans une expérience d'associations évoquées par les mots (enquête papier remplie en présentiel, site dédié tenant compte de l'exigence de faire le test en 15 minutes ou questionnaire en ligne) n'influençait pas notablement les résultats (УФимцЕВА et al 2016:6). De manière à suivre le même protocole que les expériences anglaise et russe, l'équipe du DAF a fait le choix de s'adresser uniquement à un public d'étudiants.

13 La partie informative du questionnaire contient les questions suivantes : 1) sexe, 2) âge, 3) niveau d'études, 4) domaine d'études, 5) langue maternelle (cette dernière question remplaçant une improbable et anticonstitutionnelle question sur l'origine ethnique), 6) ville où est effectué le test, 7) région. L'expérience s'est déroulée de novembre 2008 à novembre 2009, 5500 questionnaires ont été validés (500 réponses minimum par stimulus). Dans l'ensemble, on a obtenu plus de 25000 réactions dont environ 13000 ne sont pas uniques.

Le traitement ultérieur des données a porté d'une part sur l'unification des données de la partie informative (toponymes, régions, spécialisation) et sur la correction orthographique des réactions, qui n'auraient pas été exploitables dans l'état, par ex. biza, bizard, bizarrre, bizarrze, bizzar, bizzard et bizzarre pour le même adjectif. Un système de correction orthographique automatique est ici inutilisable, car dans de nombreux cas, le choix de la bonne graphie dépend du stimulus d'origine. Une forme peut ainsi correspondre à plusieurs homonymes, une réaction la pouvant être la note la, l'adjectif las, l'adverbe là et, quoique ce soit moins probable, l'article la. Pour trancher les cas litigieux, il faut tenir compte du stimulus : toto en réponse à tard doit être corrigé en tôt , par contre en réponse à résultat, il doit être réécrit totaux, tandis que la forme est correcte quand il s'agit d'une réaction au stimulus enfant. Nous sommes conscients que dans certains cas, nous n'avons peut-être pas su « deviner » le mot caché derrière une séquence énigmatique et avons donc dû éliminer quelques réactions qui auraient pu être prises en compte, mais leur nombre n'est pas important (environ une centaine sur les 25000 réactions obtenues). Notons que, étant donné le public interviewé et le fait que le questionnaire était rempli en ligne dans l'anonymat, on peut s'étonner de la quasi-absence de mots familiers, argotiques ou grossiers. Ainsi, les 4 mots grossiers 
présents parmi les 5000 plus usités de la langue française selon le dictionnaire de fréquence du français (Lonsdale 2009), soit merde, connerie, cul et putain, ne totalisent que 106 occurrences soit $0.42 \%$, fréquence comparable à celle des jeux de mots et contrepèteries (par exemple, badour comme réponse à trou parce que troubadour ou d'estal pour pied). Il convient toutefois de remarquer que la présence de calembours est particulière aux dictionnaires d'associations évoquées par les mots en français et n'a pas été remarquée dans les dictionnaires des autres langues.

Comme pour tous les dictionnaires de ce type, la structure des articles du dictionnaire direct est la suivante: l'entrée de l'article est en caractères gras, suivie par les associations, c'est-à-dire les réactions données à ce stimulus, réparties dans l'ordre inverse de fréquence. La fréquence est indiquée après chaque réaction ou à la fin d'un groupe d'associations de même fréquence, auquel cas les associations sont disposées dans l'ordre alphabétique ; les italiques indiquent les réactions qui coïncident avec des stimuli de la liste. A la fin de chaque article, on trouve quelques indications chiffrées entre parenthèses, séparées par des virgules: le nombre total de réactions à ce stimulus, le nombre de réactions différentes, le nombre de refus de réponses à ce stimulus et le nombre de réactions uniques. La structure classique d'un article de tout dictionnaire d'associations a la forme d'une hyperbole : sur 500 réactions, quelquesunes sont partagées par un grand nombre, puis cette courbe chute pour montrer un grand nombre de réactions individuelles, comme on peut le voir ici à l'article bon :

mauvais 266; bien 46; gentil 21; mal 19; délicieux 13; manger 10; bonbon, chocolat 7; agréable, beau, brute, généreux, goût, meilleur 6; juste, goutu, miam 4; brave, gâteau, méchant, nourriture, succulent 3; aimer, appétissant, bad, bonne, correct, excellent, glace, goûteux, homme, jour, savoureux, sens, sucré, trouant 2; alléchant, aloi, ange, apotropaïque, appétit, bisou, bleu, bon, bonheur, bonté, con, coup, coupon, crumble, d'achat, dégueulasse, délectable, désagréable, dessert, docile, doué, envie, fort, fruit, géant, générosité, heur, hmmmm, humain, justice, kost', marche, mieux, ok, paradis, parfait, pâtisserie, pied, plaisant, plaisir, plat, pomme, positif, pouvoir, réduction, résultat, roi, Samaritain, saveur, sentir, sexe, solde, sourire, sucre, tabac, talentueux, temps, ticket, valable, vin, vivant $1(546,97,4,61)$

Les articles du dictionnaire inverse sont formés des réactions données aux stimuli proposés lors de l'expérience. On a obtenu plus de 26000 mots, locutions, chiffres et signes typographiques (dont des émoticônes) en réponse. Les entrées des articles (en caractères gras) sont données dans l'ordre alphabétique. Chaque réaction est suivie des stimuli isolés ou réunis par groupes de même fréquence, disposés par ordre décroissant de fréquence. Le chiffre indique le nombre de personnes ayant répondu par cette réaction à ce stimulus. A l'intérieur d'un groupe, les stimuli de même fréquence sont donnés par ordre alphabétique. Ainsi, le début de l'article absence: présence 213; manquer 15, ignorer, personne, vide 4 ; signifie que la réaction absence a été donnée 213 fois au stimulus présence, 15 fois au stimulus manquer et 4 fois aux stimuli ignorer, personne, vide. A la fin de l'article sont indiqués deux chiffres entre parenthèses: il s'agit du nombre de stimuli ayant provoqué cette réaction et du nombre total de fixations de cette réaction dans le corpus. Les entrées (réactions) qui coïncident avec un stimulus de la liste d'origine sont marquées d'un astérisque. 


\subsection{Le dictionnaire des normes associatives de la Francophonie (DINAF)} francophonie s'est poursuivi par la création d'un nouveau dictionnaire, plus modeste que le précédent, car il ne porte que sur 100 stimuli. L'expérience qui a permis de réunir la matière du DINAF s'est déroulée sur internet du 15 septembre 2013 au 15 septembre 2015. Pour cela il a été proposé par l'intermédiaire d'un questionnaire Google à des locuteurs du français de France, Belgique, Suisse et Canada de participer à une expérience de fixation de la première réaction à une liste de 100 mots, identique pour tous. Nous avons évité d'y introduire des stimuli plurisémantiques pouvant porter à confusion (par ex. fin qui peut être interprété par les participants à l'expérience comme un adjectif masculin ou comme un substantif, ou marche comme un substantif, par ailleurs plurisémantique, ou une forme verbale). Dans le cas de bien, que nous avons conservé car il s'agit d'un mot très fréquent de la langue, nous n'avons pas cru bon de préciser s'il s'agissait de l'adverbe ou du substantif. Enfin, nous avons introduit quelques mots qu'il nous semblait intéressant de comparer pour différentes régions de la francophonie bien que leur fréquence ne soit pas élevée, comme, par exemple, mer. L'ordre de présentation des mots dans la liste a pour but d'éviter le voisinage de mots sémantiquement proches, ainsi que le caractère artificiel de l'ordre alphabétique.

La méthode employée pour réunir la matière a été la même pour les quatre sousensembles : à intervalles réguliers un message électronique a été envoyé vers une centaine d'adresses prises sur des sites divers, en général sur ceux d'universités francophones ou d'organismes liés à l'enseignement. Aucune restriction d'âge ou de profession n'a été imposée pour les participants à cette expérience. Un site internet a également été créé (https://sites.google.com/site/kevokcemot/), sur lequel les destinataires du message pouvaient en apprendre plus sur l'expérience, les travaux précédents et leurs résultats. Les résultats étaient collectés dans un fichier Excel. Alors que cette méthode de collecte a été strictement identique pour les quatre zones, les réponses sont arrivées dans des proportions très différentes par pays: le nombre minimal de 500 réponses a été atteint en France (notée $\mathrm{F}$ infra) au bout de six mois et a continué de croître sans actions supplémentaires de notre part, pour atteindre 604 réponses valables à la fin de l'expérience. Deux ans après le début de l'expérience et de nombreuses relances, les 500 réponses requises étaient atteintes pour la Belgique et le Canada, (resp. B et C) mais seules 326 réponses avaient été obtenues de Suisse romande (S). Nous n'avons pas d'explication à cette différence significative dans le fonctionnement de notre système de récolte des données. Nous n'avons pris en compte que les formulaires dans lesquels la partie informative très succincte, avec des questions sur le sexe, l'âge, le pays et la ville où habite la personne participant au test, ainsi que sa profession, était renseignée.

Le DINAF existe en deux versions. Dans la première, les réactions n'ont subi qu'une correction orthographique, semblable à celle décrite plus haut pour le DAF. Dans la deuxième, nous avons entrepris un travail d'unification des réactions dans le but d'en faciliter le comptage. En effet, la méthodologie traditionnelle pour les dictionnaires d'associations préconise de compter comme réactions différentes, par exemple, blanc, blancs, blanche et blanches, ce qui est justifié quand il s'agit d'analyser les relations syntagmatiques entre le stimulus et la réaction mais mène à l'apparition de quatre 
entrées différentes dans le dictionnaire inverse. De même pour les idiomes, dictons et autres locutions souvent cités en réaction sous diverses formes, par exemple c'est voler; c'est volé ; donner c'est donner, reprendre c'est voler etc. au stimulus reprendre (ДЕБРЕНН 2016). Nous nous sommes donc efforcés de présenter sous une seule forme (la plus fréquente) des réactions originellement proposées sous différentes formes. Il est prévu de faire le même travail de lemmatisation pour le DAF.

L'analyse diatopique des normes associatives avérées chez les locuteurs du français dans différentes régions de la Francophonie (Debrenne 2016) a pris en compte, pour chaque stimulus, chaque zone et en comparaison avec l'ensemble du corpus :

- les réactions les plus fréquentes, et surtout la première, la fréquence de cette réponse (en nombre absolu et en pourcentage),

- les réactions communes aux différentes zones et leur nombre (ainsi que le nombre de réactions différentes et leur quantité absolue). Ces données ont également été analysées pour l'ensemble du corpus (et non pas pour un stimulus pris à part).

- le contenu sémantique des champs associatifs (l'ensemble des réactions à un stimulus donné) suivant différentes méthodes.

21 De même, dans le dictionnaire inverse, pour chaque réaction, chaque zone et l'ensemble du corpus, nous avons comparé les stimuli qui les ont provoquées et leur nombre. Nous avons en particulier analysé pour chaque zone géographique les réactions les plus fréquentes, ainsi que celles qui ont été provoquées par le plus grand nombre de stimuli. Ces paramètres peuvent être visualisés sur le site.

A ce stade de notre étude, nous pouvons affirmer que, dans l'ensemble, quand il s'agit des réactions les plus fréquentes, les locuteurs du français des quatre zones étudiées ont tendance à réagir sensiblement de la même manière, ce qui signifierait qu'on peut parler d'une « image linguistique du monde » des locuteurs du français en général. Le fait que ces réactions soient comparables s'explique aussi par le choix des stimuli - il s'agissait de mots très courants de la langue française, partagés de manière identique par tous ses locuteurs.

Nous pensons toutefois que des différences existent dans les détails, ce qu'une analyse plus approfondie devra montrer : comme nous l'avons vu, les fréquences des réactions, même partagées par le plus grand nombre, peuvent varier d'une zone à l'autre, et, ce qui est certain, c'est que si la partition des champs sémantiques en sous-groupes est la même à chaque fois, le contenu de ces subdivisions est différent selon les différents pays. L'impression générale qui se dégage est que l'image proposée par les locuteurs du Canada est sensiblement différente de celle des "Européens " ayant participé à l'expérience.

\section{L'exploitation des données des dictionnaires d'associations évoquées par les mots}

Dans cette partie, nous donnerons quelques exemples des études menées grâce à l'utilisation de ces dictionnaires. Comme nous l'avons souligné, des recherches de ce genre sont particulièrement bien développées en Russie, en synchronie (contrastive ou non) et, depuis l'apparition du projet de nouveau dictionnaire associatif du russe, en diachronie. 


\subsection{L'analyse du champ associatif} catégories sont présentes à chaque fois, leur contenu pouvant varier d'une région à l'autre. En effet, malgré le nombre différent de réponses obtenues, les catégories déterminées pour les réponses françaises sont toutes présentes dans d'autres listes, à peu d'exceptions près (par exemple, pas d'allusions textuelles parmi les réponses canadiennes, tandis que c'est la seule liste qui propose des réactions comme portefeuille ou argent que nous avons classées - sous toutes réserves - parmi les objets fonctionnellement liées au stimulus. Seule la liste française présente une réaction «humain lié au St» (en l'occurrence architecte); seule la liste canadienne comporte une réaction hiver ( 4 - chronotope). Cependant, nous tenons à souligner que, dans l'ensemble, les quatre sous-ensembles francophones du champ associatif de maison tels que permet de l'analyser la méthode de Goldin ont beaucoup de similitudes.

\begin{tabular}{|c|c|c|c|c|c|}
\hline subcatégories & France & Belgique & Suisse & \multicolumn{2}{|l|}{ Canada } \\
\hline $\begin{array}{l}\text { 1. situation } \\
\text { avec S: }\end{array}$ & $\begin{array}{l}\text { rentre à la maison } 1 ; \\
\text { ouvrir sa maison } 1 ;\end{array}$ & $\begin{array}{l}\text { rester à la maison } \\
1 ;\end{array}$ & être à la maison 1 ; & & \\
\hline $\begin{array}{l}\text { 2. liens avec } \\
\text { S: }\end{array}$ & & & & & \\
\hline $\begin{array}{l}2.1 \\
\text { superordre }\end{array}$ & $\begin{array}{l}\text { bâtiment } 3 \text {; habitat } 9 \text {; } \\
\text { logis } 6 \text {; logement } 4 \text {; } \\
\text { immeuble } 3 \text {; pâté } 1 \text {; } \\
\text { domaine } 1 \text {; }\end{array}$ & $\begin{array}{l}\text { habitat } \\
\text { habitation } \\
\text { logement } 13 ; \\
1 ;\end{array}$ & $\begin{array}{ll}\text { habitat } & 4 ; \\
\text { habitation } & 2 ; \\
\text { immeuble 1; } & \end{array}$ & $\begin{array}{l}\text { habitat } \\
\text { immeuble } \\
\text { logis 1; }\end{array}$ & $\begin{array}{l}1 ; \\
1 ;\end{array}$ \\
\hline
\end{tabular}




\begin{tabular}{|c|c|c|c|c|}
\hline 2.2 variétés & $\begin{array}{l}\text { baraque } 1 ; \text { cabane } 4 ; \\
\text { campagne } 2 ; \text { case } 2 ; \\
\text { chaumière } 1 ; \text { close } 8 ; \\
\text { commune } 1 \text { 1; de } \\
\text { campagne } 1 ; \text { d'hôtes } 1 ; \\
\text { de retraite } 1 ; \\
\text { maisonnette } 1 \text {; pavillon } \\
2 \text {; villa } 3 ;\end{array}$ & $\begin{array}{l}\text { casa } 1 \text {; cabane } 1 \text {; } \\
\text { close } 1 ; \text { de } \\
\text { campagne } 1 \text { d de } \\
\text { retraite } 1 \text {; manoir } \\
\text { famille } 6 \text {; villa } 2 \text {; }\end{array}$ & $\begin{array}{l}\text { cabane } 1 ; \text { casa } 1 ; \\
\text { case } 1 ; \text { chalet } 2 ; \\
\text { close } 2 ; \text { cottage } 1 ; \\
\text { de vacances } 1 ; \\
\text { hutte } 1 ; \text { villa } 2 ;\end{array}$ & $\begin{array}{ll}\text { cabane } 1 ; \\
\text { chalet } 2 ; \\
\text { campagne } 1 ; \\
\text { chaumière } 2 ; \\
\text { close 4; gîte } 1 ; \\
\text { mobile 2; }\end{array}$ \\
\hline $\begin{array}{l}2.3 \text { même } \\
\text { ordre }\end{array}$ & $\begin{array}{l}\text { château } 4 ; \text { palais } 2 ; \\
\text { prison } 1 ; \text { ferme } 1 ; \\
\text { tanière } 1 ; \text { nid } ; 10 ;\end{array}$ & château 1 ; nid 9 ; & $\operatorname{nid} 1$; & tente 1 ; \\
\hline 2.4 opposé & $\begin{array}{l}\text { bateau } 2 ; \text { caverne } 1 ; \\
\text { champ } 1 ;\end{array}$ & caverne $1 ;$ mer $1 ;$ & & \\
\hline $\begin{array}{l}\text { 3. Parties de } \\
\text { S: }\end{array}$ & $\begin{array}{l}\text { appartement } 38 \text {; } \\
\text { chambre } 2 \text {; cheminée } 4 \text {; } \\
\text { cuisine } 1 ; \text { entrée } 1 ; \\
\text { fenêtre } 2 ; \text { fondation } 2 \text {; } \\
\text { garage } 2 ; \text { jardin } 13 ; \text { lit } \\
1 \text {; porte } 2 \text {; toit } 66 ;\end{array}$ & $\begin{array}{ll}\text { appartement } & 2 \text {; } \\
\text { cheminée } & 1 ; \\
\text { jardin } 3 ; \text { mur } & 1 ; \\
\text { toit } 25 & \end{array}$ & $\begin{array}{l}\text { appartement } 4 ; \\
\text { cheminée } 1 \text {; } \\
\text { garage } 1 \text {; jardin ; } \\
4 \text { : porte } 3 \text {; toit } 10 ;\end{array}$ & $\begin{array}{l}\text { chambre } 1 ; \\
\text { cheminée } 1 ; \\
\text { garage } 1 ; \text { toit } \\
11 ;\end{array}$ \\
\hline
\end{tabular}

Table №1 Fragment de l'analyse du champ associatif du stimulus maison selon la méthode de Goldin

Pour analyser le champ associatif du stimulus passer, nous avons choisi une autre méthode (celle de Goldin convenant plutôt pour les substantifs), dite de l'analyse de la signification psychologique (стЕРнин, РУдАКовА 2011 : 139). Cette méthode préconise de délimiter dans le champ associatif autant de zones de réactions liées à des significations différentes du lexème. Pour passer nous avons défini les significations suivantes: passer = mouvement (en particulier "mouvement le long de qqch", "mouvement rapide», "ce qui peut passer», «mouvement répété»); passer = franchir, traverser (en particulier "doubler, dépasser" ; passer = continuer sans s'arrêter ; passer $=$ changer d'activité (de thème de conversation) ; passer $=$ mourir ; passer = venir chez quelqu'un, éventuellement pour prendre qqch ou cette personne ; passer $=$ cesser $;$ passer $=$ occuper le temps $;$ passer $=$ transmettre $;$ passer $=$ réussir . Toutes ces catégories sont présentes dans les quatre corpus, avec, là aussi, des contenus variables en mots et en quantités : par exemple un locuteur canadien pense à une passe de hockey alors qu'un Français évoque le volley-ball. Les locuteurs des quatre zones ont également proposé des calembours basés sur l'homophonie passer / passé: nous trouvons dans chaque corpus un présent, un futur et même un composé.

\begin{tabular}{|l|l|l|l|l|}
\hline passer & & & & \\
\hline signification & France & Belgique & Suisse & Canada \\
\hline
\end{tabular}




\begin{tabular}{|c|c|c|c|c|}
\hline spec mouvement répété & $\begin{array}{l}\text { encore, } 2 \\
\text { et repasser } 1 \\
\text { repasser } 16 \\
\text { revenir } 3\end{array}$ & $\begin{array}{l}\text { repasser } 5 \\
\text { revenir } 1\end{array}$ & $\begin{array}{l}\text { cyclique } 1 \\
\text { repasser } 3 \\
\text { revenir } 3 \\
\text { souvent }\end{array}$ & $\begin{array}{l}\text { repasser } 3 \\
\text { revenir } 1\end{array}$ \\
\hline $\begin{array}{l}\text { passer }=\text { franchir, } \\
\text { traverser }\end{array}$ & $\begin{array}{l}\text { de l'autre côté } 1 \\
\text { à travers } 3 \text {; } \\
\text { étape } 1 \\
\text { franchir } 2 \\
\text { frontière } 1 \\
\text { fenêtre } 1 \\
\text { de l'autre côté de la } \\
\text { barrière } 1 \\
\text { le pont } 1, \\
\text { un pont } 2 \\
\text { outre } 14 \\
\text { outrepasser } 1 \\
\text { transiter } 1 \\
\text { traverser } 18 \\
\text { laisser } 16 \\
\text { laisser-passer } 1\end{array}$ & $\begin{array}{l}\text { cap 1 } \\
\text { frontière 1 } \\
\text { la porte 2, } \\
\text { porte } 1 \\
\text { outre } 3 \\
\text { passage } 1 \\
\text { passage piéton } 1 \\
\text { traverser } 5 \\
\text { laisser } 4\end{array}$ & $\begin{array}{l}\text { le gué } 1 \\
\text { le pont } 1 \\
\text { pont } 1 \\
\text { un pont } 1 \\
\text { outre } \\
\text { rive } 1 \\
\text { traverser } 5 \\
\text { laisser } 2\end{array}$ & $\begin{array}{l}\text { la rampe } 1 \\
\text { laissez-passer } \\
1 \\
\text { laisser } 1 \\
\text { outre } 10 \\
\text { passer outre } 1 \\
\text { traverser } 3 \\
\text { transition } 1\end{array}$ \\
\hline spec. passer $=$ doubler & $\begin{array}{l}\text { devant } 5 \\
\text { dépasser } 5\end{array}$ & $\begin{array}{l}\text { dépasser } 1 \\
\text { devant } 1\end{array}$ & devant 2 & $\begin{array}{l}\text { dépasser } 1 \\
\text { devant } 5\end{array}$ \\
\hline $\begin{array}{l}\text { passer = continuer sans } \\
\text { s'arrêter }\end{array}$ & $\begin{array}{l}\text { chemin } 15, \\
\text { passer son chemin } 2, \\
\text { son chemin } 7, \\
\text { continuer } 2 \\
\text { ne pas s'arrêter } 3 \\
\text { ne pas s'installer } 1 \\
\text { nomade } 1 \\
\text { sans s'arrêter } 2 \\
\text { s'arrêter } 1 \\
\text { rester } 10\end{array}$ & $\begin{array}{l}\text { chemin } 2 \\
\text { passer } \\
\text { chemin } 1 \\
\text { son chemin } 4 \\
\text { rester 7 } \\
\text { s'arrêter 2 }\end{array}$ & $\begin{array}{l}\text { chemin } 1 \\
\text { son chemin } \\
5 \\
\text { survoler } 1 \\
\text { transit } 1 \\
\text { rester } 6\end{array}$ & $\begin{array}{l}\text { chemin } 1 \\
\text { ne pas } \\
\text { s'arrêter 1 } \\
\text { poursuivre 1 } \\
\text { rester 7 }\end{array}$ \\
\hline
\end{tabular}

Table №2 Fragment de l'analyse du champ associatif du stimulus passer selon la méthode de Sternin

Enfin pour le stimulus noir, nous avons utilisé la méthode dite de la Gestalt sémantique, (КАРАУЛОВ, 2000 : 194). C'est, de loin, la méthode la plus fréquemment utilisée en Russie pour l'analyse des champs associatifs; elle est assez intuitive puisqu'elle préconise pour chaque champ de définir environ 5-7 sous-ensembles: " une Gestalt associative est mise en évidence quand les associations tendent vers des caractéristiques en se regroupant autour de quelques réactions (en général les plus fréquentes) qui définissent ou nomment un ensemble d'images mentales, les concepts. 
Ces concepts signalent de la manière la plus générale les liens typiques du référent désigné dans la culture en question par le stimulus, c'est pourquoi on peut utiliser la Gestalt sémantique pour la comparaison interculturelle des données associatives " (МАРКОВИНА, ДАНИЛОВА 2000 : 119).

Dans le cas du stimulus noir (qui pouvait indifféremment être interprété par les participants à l'expérience comme un adjectif ou un substantif), les réactions associatives se sont réparties selon les zones suivantes: 1 - couleur (avec une forte préférence pour blanc comme antonyme) ; 2 - objets de cette couleur ; 3 - obscurité, 4 sentiments évoqués; 5 - couleur de peau; On peut également déterminer quelques autres sous-zones plus restreintes : 6 - habits de couleur noire ; 7 - idée liée à la mort, ainsi que les inclassables "allusions textuelles » et jeux de mots, notamment d'assez fréquents noir c'est noir ou Désir. Là aussi nous retrouvons sensiblement les mêmes zones pour les quatre sous-ensembles étudiés, avec cependant des différences de contenu. Seuls les interrogés français ont évoqué l'ivresse (réactions comme un Polonais, ivre). De même nous n'avons trouvé des émotions positives que dans le corpus français : positif ou négatif, vie et mort ; protection ; rassurant etc.

\begin{tabular}{|c|c|c|c|c|}
\hline concepts & France & Belgique & Suisse & Canada \\
\hline $\begin{array}{l}\text { homme de race } \\
\text { noire }\end{array}$ & $\begin{array}{l}\text { africain } 1 \\
\text { homme } 1 \\
\text { homme noir } 1 \\
\text { racisme } 1\end{array}$ & $\begin{array}{l}\text { africain } 1 \\
\text { Afrique } 1 \\
\text { homme } 1\end{array}$ & & diversité 1 \\
\hline ivresse & $\begin{array}{l}\text { comme un Polonais } 1 \\
\text { ivre } 1\end{array}$ & & & \\
\hline jeux de mots & croire & & & \\
\hline $\begin{array}{l}\text { allusions } \\
\text { intertextuelles }\end{array}$ & $\begin{array}{l}\text { c'est noir } 5 \\
\text { noir c'est noir } 1 \\
\text { noir c'est noir il n'y a plus } \\
\text { d'espoir } 1 \\
\text { Désir } 4 \\
\text { is beautiful } 1 \\
\text { rouge } 3\end{array}$ & $\begin{array}{l}\text { noir c'est } \\
\text { noir } 1 \\
\text { de monde } 1 \\
\text { Désir } 5 \\
\text { neige } 1 \\
\text { Etoile } 1\end{array}$ & $\begin{array}{l}\text { Désir } \\
\text { rouge } 4 \\
\text { John Howard } \\
\text { Griffin } 1 \\
\text { l'oiseau noir } 1 \\
\text { piano } 1\end{array}$ & rouge 2 \\
\hline $\begin{array}{l}\text { habits de couleur } \\
\text { noire }\end{array}$ & $\begin{array}{l}\text { amincit } 1 \\
\text { chic } 3 \\
\text { classe } 2 \\
\text { discrétion } 1 \\
\text { élégance } 1 \\
\text { joli } 1 \\
\text { minceur } 1 \\
\text { gothique } 1\end{array}$ & $\begin{array}{l}\text { élégance } 2 \\
\text { manteau } 1 \\
\text { vêtement } 1\end{array}$ & élégance 2 & $\begin{array}{l}\text { classique } 1 \\
\text { costume } 1 \\
\text { élégant } 1 \\
\text { habillement } \\
1 \\
\text { mode } 1\end{array}$ \\
\hline
\end{tabular}




\begin{tabular}{|l|l|l|l|l|}
\hline mort & $\begin{array}{l}\text { deuil 6 } \\
\text { funérailles 1 }\end{array}$ & $\begin{array}{l}\text { deuil 3 } \\
\text { mort }\end{array}$ & $\begin{array}{l}\text { deuil 3 } \\
\text { enterrement 1 }\end{array}$ & mort 1 \\
\hline
\end{tabular}

Table №3 Fragment de l'analyse du champ associatif du stimulus noir selon la méthode de Karaulov

\subsection{Les superconnecteurs}

31 L'analyse des données du dictionnaire inverse permet d'établir la liste des réactions liées au plus grand nombre de stimuli. Cette liste, désignée par les psycholinguistes russes comme «noyau de la conscience linguistique », représentative de la structure en réseau du lexique, est présentée dans l'ordre décroissant du nombre de stimuli. Les premières réactions de cette liste, liées à des centaines de stimuli, et interconnectées entre elles, sont les superconnecteurs du «small world» du lexique. Des notions analogues sont développées dans les travaux d'A. Polguère sur les réseaux lexicaux (Polguère 2014). Ce sont également les mots les plus riches en connotations d'une langue donnée.

Ci-dessous on peut voir les listes établies d'après les données du DINAF :

\begin{tabular}{|c|c|c|c|c|c|}
\hline & $\begin{array}{l}\text { France (sur } 100 \\
\text { stimuli) }\end{array}$ & $\begin{array}{l}\text { Belgique (sur } 100 \\
\text { stimuli) }\end{array}$ & $\begin{array}{l}\text { Suisse (sur } 100 \\
\text { stimuli) }\end{array}$ & $\begin{array}{l}\text { Canada (sur } 100 \\
\text { stimuli) }\end{array}$ & $\begin{array}{l}\text { France-2010 (sur } \\
1100)\end{array}$ \\
\hline 1 & vie 48 & vie 39 & vie 28 & vie 39 & vie 315 \\
\hline 2 & vivre 45 & bien 32 & vivre 27 & bien 32 & amour 245 \\
\hline 3 & être 35 & être 31 & être 24 & amour 29 & homme 193 \\
\hline 4 & bien 32 & vivre 28 & aimer 21 & être 27 & bien 166 \\
\hline 5 & aimer 31 & amour 27 & bon 21 & plaisir 27 & mort 164 \\
\hline 6 & dire 30 & bonheur 26 & amour 20 & beau 25 & bonheur 154 \\
\hline 7 & grand 28 & beau 23 & beau 19 & espoir 24 & travail 154 \\
\hline 8 & beau 27 & grand 22 & bien 18 & joie 24 & joie 153 \\
\hline 9 & amour 27 & temps 21 & grand 18 & bonheur 24 & enfant 152 \\
\hline 10 & espoir 26 & mort 21 & mal 18 & partir 22 & noir 151 \\
\hline 11 & temps 26 & lumière 20 & fort 18 & tout 22 & temps 146 \\
\hline 12 & mal 25 & bleu 20 & savoir 17 & rien 22 & aimer 144 \\
\hline 13 & bonheur 25 & aimer 19 & femme 17 & beauté 22 & mal 144 \\
\hline 14 & enfant 23 & partir 19 & temps 16 & bon 21 & plaisir 144 \\
\hline
\end{tabular}




\begin{tabular}{|l|l|l|l|l|l|}
\hline 15 & penser 22 & dire 18 & partir 16 & mal 21 & droit 142 \\
\hline 16 & homme 22 & plaisir 18 & enfant 16 & savoir 21 & vivre 139 \\
\hline 17 & plaisir 22 & prendre 18 & espoir 16 & enfant 21 & personne 138 \\
\hline 18 & prendre 22 & savoir 18 & liberté 16 & mort 21 & peur 136 \\
\hline 19 & savoir 22 & loin 18 & manger 16 & temps 20 & terre 136 \\
\hline 20 & personne 21 & enfant 17 & homme 15 & penser 20 & fin 135 \\
\hline 21 & libre 21 & espoir 16 & joie 15 & cœur 20 & liberté 134 \\
\hline 22 & joie 20 & faire 16 & penser 15 & vivre 19 & ami 133 \\
\hline 23 & faire 20 & donner 16 & libre 15 & aimer 19 & argent 133 \\
\hline 24 & rien 20 & rester 16 & bonheur 14 & grand 19 & donner 133 \\
\hline 25 & toujours 20 & rêve 16 & plaisir 14 & donner 19 & moi 133 \\
\hline
\end{tabular}

Table № 4 Liste comparative des 25 réactions liées au plus grand nombre de stimuli dans les dictionnaires d'association du français. Sont indiqués la réaction et le nombre de stimuli qui l'ont provoquée. La dernière colonne présente les données obtenues dans le DAF en 2010 pour comparaison

Dans chaque case du tableau sont portés la réaction et le nombre de stimuli pour lesquels cette réaction a été proposée au moins une fois. Par exemple, pour les participants de France, la réaction vie figure dans les champs associatifs de presque la moitié des stimuli de la liste (48), avec une fréquence totale de 369 occurrences. Parmi les locuteurs des autres pays, cette réaction est également celle qui est provoquée par le plus grand nombre de stimuli, dans une proportion moindre, mais avoisinant ou dépassant le tiers des stimuli. C'était aussi le cas pour les étudiants qui ont participé à l'expérience associative en 2008-2009. La liste des stimuli qui ont provoqué l'apparition de la réaction vie et sa fréquence varie selon les zones étudiées de la francophonie, cependant 18 stimuli sont communs aux quatre: il s'agit de mort, coeur, amour, vivre, aimer, reprendre, âme, air, jeune, mourir, soleil, corps, donner, ami, comprendre, enfant, gens, devenir (dans l'ordre décroissant du nombre d'occurrences de cette réaction dans le corpus).

L'utilisation des listes de superconnecteurs pour telle ou telle langue permet de compléter l'analyse linguistique traditionnelle et d'observer les divergences culturelles du point de vue du caractère systémique du modèle du monde d'une culture donnée. C'est seulement ainsi que nous pouvons nous faire une idée de l'importance réelle (du point de vue d'un représentant d'une culture donnée) ou du manque d'importance des divergences mises en évidence. Envisageons l'objet culturel qu'est un ami.

Analysant le contenu des concepts AMITIÉ ${ }^{2}$ et AMI, A. Wierzbicka aboutit à la conclusion que l'assertion selon laquelle "dans toutes les cultures "les amis" sont considérés comme catégorie psychologique et sociale importante » est fausse. En ce qui concerne la culture anglaise le sens et le fond de l'idée d'AMITIÉ et d'AMI se sont 
essentiellement dévalués durant le temps. Comme l'affirme A. Wierzbicka, «Broadly, the meaning of the word friend has "weakened," so that to achieve anything like the same "force" it is now necessary to use the expression close friend. Something of the old value of the word friend has survived in the derived noun friendship: whereas in the older usage, friends were related to one another by friendship, in the current usage one can have many more friends than friendships, and only "close friends" can now be said to be linked by "friendship."» (Wierzbicka 1997: 36).

Wierzbicka indiquait que, dans la culture anglo-américaine, l'idée de "faire connaissance de nouvelles personnes" a pris la place de celle de "établir des liens d'amitié" comme un rapport constant entre les personnes. Dans cette culture, ces personnes s'appellent des "amis". Par contre, selon N. M. Lebedeva, la protection de la famille, la santé, l'amitié vraie, la fidélité, l'intellect, le sens de la vie, l'harmonie intérieure font partie des valeurs de base de la culture russe et elles se transmettent de génération en génération (ЛЕБЕДЕВА 2000).

Considérons maintenant les données des dictionnaires associatifs. D’après le Dictionnaire associatif russe indirect, la notion d'ami, donnée comme réaction à 565 stimuli différents et occupant le $9^{\text {ème }}$ rang dans le noyau, tient une place très importante dans le noyau de la conscience linguistique des Russes. Le champ associatif de ДРуг (ami) montre qu'il est avant tout ВЕРНЫЙ (fidèle 69 fois), НАДЕЖНЫЙ (sûr 9), НАСТоЯщИЙ (vrai 9), СТАРЫЙ (vieux 4). D’autres caractéristiques importantes de l'ami sont БлИзКИй (proche 16), люБИмЫЙ (aimé 4), СЕРДЕчныЙ (cordial 4). Il est naturel qu'un tel ami soit лучший (le meilleur 20), люБЕзный (aimable 12), Единственный (unique 9), c'est un ami ДЕТСВТА (d'enfance 33). L'ami est тОВАРИщ (camarade 27) et БРАТ (frère 10), mais aussi, très souvent, c'est un СОБАКА (chien 17). L'opposition ami - ВРАГ (ennemi 47), bien qu'elle soit permanente dans la conscience des Russes contemporains, est présentée en général par un groupe de réactions très peu nombreux dans le champ associatif du stimulus ami. L'importance extraordinaire de l'ami dans la vie de l'homme russe révèle le fait que, d'après les données du dictionnaire de N.I. Beresneva, L. A. Doubovskaya, I. G. Otchinnikova (БЕРеCHEBA et al. 1995), on voit que dès l'âge de dix ans, l'ami occupe une place très importante dans le modèle du monde des enfants élevés dans la culture russe. C'est prouvé par le fait que le mot ami se rencontre dans leur dictionnaire comme réaction 568 fois sur 33 stimuli présentés à des enfants dans un test d'associations spécifique, ce qui est confirmé par les données de Goldin pour Saratov (гольдин et al 2004).

Si on analyse de plus près les données du Dictionnaire inverse, on voit que le mot ami apparaitt le plus souvent comme réaction aux stimuli нАстоящий (vrai, 215), лучший (meilleur 156), ВЕРНЫЙ (fidèle 64), НАДЕЖНЫЙ (sûr 52).

Dans le modèle du monde des locuteurs de l'anglais, selon Kiss, nous obtenons une image tout à fait différente. La notion friend occupe la $73^{3 \text { ème }}$ place dans le noyau de leur conscience linguistique et se distingue par une couleur émotionnelle tout à fait différente. Dans le dictionnaire direct (Kiss et al 1972) friend est associé avant tout avec enemy (22), foe (19), suivi par girl (4) et good (4). D'après les données du Dictionnaire inverse la réaction friend apparaît le plus souvent à des stimuli comme acquaintance (68)+ acquuaintance $\left(67^{3}\right.$, companion (67), college (50), buddy (36), neighbor (36)+ neighbourg (33), ally (35). 
Apparaissant en réaction à 133 mots différents, ami occupe dans le noyau de la conscience linguistique des Français la $22^{\text {ème }}$ place, à laquelle il faut ajouter celle d'amis, cité 104 fois comme réaction (ДЕБРенн 2010a); c'est une place assez importante. Dans le dictionnaire direct, on constate que, pour les Français, un ami est associé tout d'abord à copain (82) ou pote (16), et presque deux fois moins souvent, à ennemi (54); il est fidèle (15); proche (12), c'est le meilleur (8); les sentiments qu'on éprouve pour lui sont de l'amitié (18) ou de l'amour (16). Dans le dictionnaire inverse, on constate (ДЕБРенН 2010 b) que la réaction ami apparaît le plus souvent à compagnon (544), camarade (213), ennemi (185), conseil (78), confiance (64), un voisin (61), compagnon (50) confier (29).

41 Ajoutons que, d'après les données du DINAF, et ce, malgré la popularité des réseaux sociaux et de la notion d'ami qu'ils véhiculent, cette réaction a fortement reculé et n'occupe plus que la $35^{\text {ème }}$ place au Canada, la $53^{\text {ème }}$ en France, la $69^{\text {ème }}$ en Belgique et la 72 ème en Suisse. Ainsi, tout en restant dans le "noyau de la conscience linguistique", limité arbitrairement aux 75 premières réactions, cette notion semble avoir perdu de son importance aux yeux des locuteurs du français. Nous tenons à souligner cependant qu'à nos yeux les dictionnaires d'associations évoquées par les mots sont des instruments de mesure: ils mettent en évidence des tendances. Pour respecter les principes de rigueur scientifique, ces mesures doivent impérativement être répétées. Elles ne doivent en aucun cas être utilisées pour des interprétations hasardeuses et forcément réductrices de la mentalité des locuteurs. Pour cette raison le projet d'une nouvelle édition du dictionnaire des associations évoquées par les mots a été lancé en 2018 (Debrenne 2018). Il tiendra compte notamment des écueils rencontrés dans la réalisation du précédent et du changement des mentalités, notamment parmi les interviewés. Actuellement, on peut suivre le déroulement de la collecte de données et les premiers résultats sur internet. Depuis la première expérimentation, dix ans ont passé et une nouvelle permettra de comparer, dans une perspective diachronique, ce qui est stable et ce qui est éphémère dans les champs d'associations.

\subsection{Applications dans l'enseignement du FLE}

Notons pour conclure ce développement que les dictionnaires d'associations évoquées par les mots peuvent être d'une grande utilité dans l'étude d'une langue étrangère. Par exemple, l'analyse du réseau associatif verbal révélé par le dictionnaire inverse permet de compléter l'information fournie par un dictionnaire de fréquence pour repérer les mots à enseigner en premier lieu. On peut également analyser avec les apprenants le contenu sémantique d'une notion et surtout la charge culturelle partagée. Un grand nombre de réactions sont conditionnées par des connaissances de "culture générale " présentée notamment (mais pas exclusivement) par les noms propres donnés en réaction aux stimuli de la liste d'origine. Sur 26000 réponses, on a obtenu 1500 noms propres, soit $6 \%$ (sans compter les gentilés, les sigles, les corps célestes et les noms propres contenus dans un syntagme type " œuf de Pâques ", "à la grâce de Dieu »). Les noms propres sont des toponymes (continents, pays, régions, villes, noms de rues, places, monuments), des indications d'événements historiques ou contemporains, de fêtes, des noms de personnalités (architectes, écrivains, peintres, philosophes, acteurs, metteurs en scène, musiciens, compositeurs, chanteurs; personnages historiques, hommes politiques, sportifs, " people »), titres d'œuvres d'art, sculpture, musique, films, ou jeux vidéo, des noms de personnages, noms de marque, d'institutions, des 
termes de religion ou de mythologie, des artefacts et termes scientifiques, des prénoms, ou des noms de famille. On pourrait créer un glossaire culturel explicitant ces allusions (par exemple des associations telles président $\rightarrow$ Zébulon, sœur $\rightarrow$ Emmanuelle ou gens $\rightarrow$ Gabin) non seulement pour les étrangers, mais aussi pour les Français qui, d'ici quelques années pourraient oublier quelques références liées à l'actualité (par exemple boubou $\rightarrow$ Ségolène à Dakar, allusion à un épisode anecdotique de la campagne présidentielle de 2007).

\section{Conclusion}

43 Le DINAF a été entrepris notamment pour apporter une réponse la plus objective possible à la question de l'existence d'une "vision du monde » soutenue par la langue, dite également «image linguistique du monde » (ILM) autour de laquelle se déroulent de vifs débats (Pycco 2012) et qui peut être définie ainsi : "La sémantique des unités lexicales comprend un certain nombre de " présomptions ", c'est-à-dire de représentations du monde non reflétées dans les usages assertifs courants de cette unité et qui passent souvent inaperçus des participants de la communication (présupposés, connotations, composants de base de la signification). [...] Au cours de la communication ces présomptions ne sont généralement pas mises en doute, et un emploi de cette unité de langue en contradiction avec elles provoque une réaction négative des communicants [...] C'est précisément de l'ensemble de ces présomptions que se compose l'image (le modèle) linguistique du monde. » (шмЕлЕв 2011) Comme on le voit, il s'agit donc ici uniquement de la structure du champ sémantique des mots, et il convient de distinguer l'ILM telle qu'elle est mise en évidence par les dictionnaires d'associations des objets étudiés par la linguistique cognitive (les concepts, le « lexique mental » etc.).

44 Nous soutenons que l'étude des associations évoquées par les mots est une méthode adéquate et relativement objective d'étude des connotations sous-tendues par les mots. Ainsi, si les locuteurs d'une même langue se trouvant dans des conditions sociolinguistiques différentes (tels, dans le cas du DINAF, les Belges et les Canadiens) présentent néanmoins des réactions d'association semblables, on peut en conclure que cette image de la langue dépend de la langue donnée et non pas des conditions de l'environnement. On sait que, par ailleurs, ces associations sont sensiblement différentes dans une même langue à différentes époques, mais également d'une langue à l'autre, donc, là aussi, on peut penser qu'il y a un lien entre ILM et langue (УФимЦЕВА 2013).

45 Cette méthode a bien sûr ses limites: d'une part, jusqu'à présent, les expériences d'associations lexicales ne prenaient en compte qu'un nombre limité de réponses, faisant de leur validité un éternel sujet de querelle entre psycholinguistes et sociolinguistes. A l'heure actuelle, grâce à internet, cette difficulté peut être surmontée, et plus on aura de participants, plus l'analyse sera objective. Par ailleurs, la difficulté réside dans le degré de précision de notre analyse: comme on l'a vu, la structure d'un champ associatif comporte une petite quantité d'associations communes à un grand nombre, puis un grand nombre d'associations uniques. Cette structure, comme dans le cas de l'optimiste et du pessimiste devant un verre à moitié plein (vide), peut être interprétée de deux manières: pour les uns la grande fréquence des premières réactions prouve que la plupart des interviewés réagissent de la même 
manière, ce qui démontre l'existence de l'ILM. Pour les autres le fait qu'une bonne moitié des réactions soient des hapax infirme cette hypothèse. Nous avons cependant vu plus haut qu'on pouvait analyser plus finement les réactions uniques en les regroupant par groupes sémantiques, ce qui permet de confirmer la première hypothèse plutôt que la seconde. Une troisième difficulté réside, à notre avis, dans l'interprétation des résultats obtenus. Très populaires en Russie et utilisés dans les recherches à tous niveaux, les dictionnaires d'associations évoquées par les mots donnent lieu trop souvent à des généralisations trop hâtives ou injustifiées au vu de l'ampleur de l'étude réalisée. Au lieu d'affirmer qu'ils permettent de " mettre en évidence la conscience linguistique des Français " (par exemple) il faudrait écrire qu'ils montrent "les normes associatives des participants à l'expérience ». Trop souvent des expressions telles « le dictionnaire associatif russe » ou « l'image linguistique russe du monde » sont perçues comme "le dictionnaire des associations verbales des Russes (ethniques) » et non pas «de la langue russe " ou "des locuteurs du russe ». Si l'on analyse l'immense quantité de recherches publiées en Russie ces dernières années à propos de l'ILM ou des "concepts", on peut y trouver des formulations douteuses telle "la langue nationale forme le caractère national ", ce qui a été relevé à plusieurs reprises (АПРЕСЯН 2006: 35, ПАВЛОВА 2009, 2010). De même, nous considérons comme abusives des conclusions sur la "westernisation de la mentalité russe" basées sur la présence d'anglicismes dans les réponses des étudiants sibériens (ШАПошниковА 2016).

\section{BIBLIOGRAPHIE}

Debrenne M. Variabilité diatopique des associations évoquées par les mots en Francophonie // 5me congrès Mondial de Linguistique Française Tours 4-8 juillet 2016 ; DOI 10.1051/shsconf/ 20162703002

Debrenne M. La lexicographie associative : vers un nouveau dictionnaire français des associations évoquées par les mots // SHS Web Conf. Volume 46, 2018 6e Congrès Mondial de Linguistique Française. DOI 10.1051/shsconf/20184605005

Grundlagen der sprachlichen Analogiebildung // Revue Philosophique de la France et de l'Étranger 1901, 52 : 559-560.

Hagège C. (2012) Contre la pensée unique. Paris : O. Jacob, 2012

Kent, G. H., \& Rosanoff, A. J. (1910). ... American Journal of Insanity 67, 37-96, 317-390.

Kiss G., Armstrong C., Milroy K. The Associative Thesaurus of English, Edinburg: Univ. of Edinb. (MGC Speech and Communication Unit), 1972.

Lonsdale, Le Bras, A Frequency Dictionary of French, Core vocabulary for learners, London, New York 2009.

Nelson, D. L., McEvoy, C. L., \& Schreiber, T. A. (1998). The University of South Florida word association, rhyme, and word fragment norms. http://www.usf.edu/FreeAssociation. 
Peeters B. (2013) La langue de bois : un pèlerinage ethnolexicologique. Marillaud Pierre, Gauthier Robert (éds), La mauvaise parole, 33e Colloque d'Albi Langages et Signification, Albi / Toulouse, CALS/CPST, pp. 196-210.

Polguère A. (2014) From Writing Dictionaries to Weaving Lexical Networks. International Journal of Lexicography 27(4), pp. 396-418.

Postman L. (1970). Norms in word association. N.Y.: Academic Press.

Puig S., Karaulov Yu., Cherkasova G. (2001). Normas asociativas del espagnol y del ruso). M., МАДРИд : АЗБУКОВНИк, 2001.

Raccah, P.-Y. (2014). Sémantique des points de vue et contraintes sur les possibles argumentatifs. Du sens à la signification. De la signification aux sens, Bruxelles, New York ; Peter Lang ; pp. 277-300

Russell, W. A., \& Jenkins, J. J. The complete Minnesota norms for responses to 100 words from the Kent-Rosanoff word association test. Technical Report No. 11. Contract N8-ONR-66216, ONR. Minneapolis: Univ. Minnesota, 1954.

Thumb A., Marbe K. Experimentelle Untersuchungen über die psychologischen Underhill, J. (2012). Ethnolinguistics and cultural concepts, Truth, Love, Hate and War. Cambridge: Cambridge University Press. Deryle Lonsdale, Yvon le Bras (2009)

Wierzbicka A. Understanding Cultures Through Their Key Words. New York Oxford. Oxford University Press, 1997, 285 p.

АПРЕСЯН Ю. Д. ОСНОВАНИЯ СИСТЕМНОЙ ЛЕКСИКОГРАФИИ // ЯЗЫКОВАЯ КАРТИНА МИРА И СИСТЕМНАЯ ЛЕКСИКОГРАФИЯ. М., 2006.

БЕРЕСНЕВА Н. И., ДУБОВСКАЯ Л. А., ОВЧИННИКОВА И. Г. АССОЦИАЦИИ ДЕТЕЙ ОТ ШЕСТИ ДО ДЕСЯТИ ЛЕТ, ПЕРМЬ, 1995.

ВЕЖБИЦКАЯ А. ПОНИМАНИЕ КУЛЬТУР ЧЕРЕЗ ПОСРЕДСТВО КЛЮЧЕВЫХ СЛОВ. МОСКВА (ЯЗЫКИ слАВянскоЙ кУЛЬТУРЫ), 2001.

ГОЛЬДИН В. Е., МАРТЬЯНОВ А. О., СДОБНОВА А. П. «АССОЦИАТИВНЫЙ СЛОВАРЬ ШКОЛЬНИКОВ САРАТОВА И САРАТОВСКОЙ ОБЛАСТИ» in ЯЗЫКОВОЕ СОЗНАНИЕ: ТЕОРЕТИЧЕСКИЕ И ПРИКЛАДНЫЕ АСПЕКТЫ, УФИМЦЕВОЙ Н.В. (РЕД), МОСКВА-БАРНАУЛ, 2004, С. 215 - 226.

ГОЛЬДИН, В. Е., (2010). КОНЦЕПТУАЛЬНЫЕ ПЕРЕМЕННЫЕ ОБРАЗА МИРА ПО ДАННЫМ АССОЦИАТИВНЫХ СЛОВАРЕЙ , КОМПЬЮТЕРНАЯ ЛИНГВИСТИКА И ИНТЕЛЛЕКТУАЛЬНЫЕ ТЕХНОЛОГИИ. ВЫП. 9 (16). сс. 97-100.

ГОЛЬДИН, В. Е., МАРТЬЯНОВ, А. О., СДОБНОВА, А. П. (2009) ЭЛЕКТРОННЫЙ РУССКИЙ АССОЦИАТИВНЫЙ СЛОВАРЬ ШКОЛЬНИКОВ, КОМПЬЮТЕРНАЯ ЛИНГВИСТИКА И ИНТЕЛЛЕКТУАЛЬНЫЕ ТЕХНОЛОГИИ. ВЫП. 8 (15). С. 69-74.

ГУЦ Е.Н. АССОЦИАТИВНЫЙ СЛОВАРЬ ПОДРОСТКА, ОМСК, 2004

ДЕБРЕНН М. ЛЕКСИЧЕСКИЕ ОТНОШЕНИЯ В АССОЦИАТИВНЫХ СЛОВАРЯХ ФРАНЦУЗСКОГО ЯЗЫКА // вопросы психолингвИстики 2016, Вып. 1, №16 с.76-89

КАРАУЛОВ Ю. Н., СОРОКИН Ю. А., ТАРАСОВ Е. Ф., УФИМЦЕВА Н. В., ЧЕРКАСОВА Г. А. РУССКИЙ АССОЦИАТИВНЫЙ СЛОВАРЬ. Т. Т.1-6, М.: ИНСТИТУТ РУССКОГО ЯЗЫКА РАН, 1994-1998. 
КАРАУЛОВ, Ю. Н. (2000). ПОКАЗАТЕЛИ НАЦИОНАЛЬНОГО МЕНТАЛИТЕТА В АССОЦИАТИВНОВЕРБАЛЬНОЙ ЦЕПИ. ЯЗЫКОВОЕ СОЗНАНИЕ И ОБРАЗ МИРА., МОСКВА: ИН-Т ЯЗЫКОЗНАНИЯ РАН. СС. 191-206.

ЛЕБЕДЕВА Н. «БАЗОВЫЕ ЦЕННОСТИ РУССКОЙ КУЛЬТУРЫ НА РУБЕЖЕ ХХ1 ВЕКА», in ПСИХОЛОГИЧЕСКИЙ ЖУРНАЛ, Т. 21, № 3, 2000, с. 73-87.

ЛЕОНТЬЕВ А. А. (1993) «ЯЗЫКОВОЕ СОЗНАНИЕ И ОБРАЗ МИРА», in ЯЗЫК И СОЗНАНИЕ:

ПАРАДОКСАЛЬНАЯ РАЦИОНАЛЬНОСТЬ, МОСКВА, С.16-21.

ЛЕОНТЬЕВ А. А. (1997) ОСНОВЫ ПСИХОЛИНГВИСТИКИ, МОСКВА, СМЫСЛ, 1997

ПАВЛОВА А. В. ЛЕКСИЧЕСКАЯ ЛАКУНАРНОСТЬ И ГИПОТЕЗА ЛИНГВИСТИЧЕСКОЙ

ОТНОСИТЕЛЬНОСТИ // РУССКАЯ ГЕРМАНИСТИКА: ЕЖЕГОДНИК. М., 2009. Т. 6. С. 254-259.

ПАВЛОВА А., БЕЗРОДНЫЙ М. ЛОЖНЫЙ ВЫзОВ // Toronto Slavic Quarterly. 2010. № 34. URL:

http://www.utoronto.ca/.

ПАВЛОВА А., БЕЗРОДНЫЙ М. ХИТРУШКИ И ЕДИНОРОГ: ОБРАЗ РУССКОГО ЯЗЫКА ОТ ЛОМОНОСОВА ДО ВЕЖБИЦКОЙ // Toronto Slavic Quarterly.2010. № 32. URL: http://www.utoronto.ca/.

РУДАКОВА А.В., КОВАЛЕНКО С.В., СТЕРНИН И. А. ПСИХОЛИНГВИСТИЧЕСКИЙ ТОЛКОВЫЙ СЛОВАРЬ РУССКОГО ЯЗЫКА. ВЫПУСК 1. АНТРОПОНИМЫ ВОРОНЕЖ 2018

РУДАКОВА А.В., КОВАЛЕНКО С.В., СТЕРНИН И. А. ПСИХОЛИНГВИСТИЧЕСКИЙ ТОЛКОВЫЙ СЛОВАРЬ РУССКОГО ЯЗЫКА. ВЫПУСК 2. АНТРОПОНИМЫ ВОРОНЕЖ 2018127 СТР.

РУССКИЙ АССОЦИАТИВНЫЙ СЛОВАРЬ. В 2 Т. / Ю.Н.КАРАУЛОВ, Г.А. ЧЕРКАСОВА, Н.В. УФИМЦЕВА, Ю.А. СОРОКИН, Е.Ф. ТАРАСОВ. Т. І. ОТ СТИМУЛА К РЕКЦИИ: ОК. 7000 СТИМУЛОВ. М.: АСТ-АСТРЕЛЬ, 2002. 784 С. Т. ІІ. ОТ СТИМУЛА К РЕКЦИИ: БОЛЕЕ 100000 РЕАКЦИЙ. М.: АСТ-АСТРЕЛЬ, 2002.992

РУССКИЙ РЕГИОНАЛЬНЫЙ АССОЦИАТИВНЫЙ СЛОВАРЬ-ТЕЗАУРУС ЕВРАС, ТОМ 1, ОТ СТИМУЛА К РЕАКЦИИ, ЧЕРКАСОВА Г.А., УФИМЦЕВА Н.В., 2014.

РУССКИЙ РЕГИОНАЛЬНЫЙ АССОЦИАТИВНЫЙ СЛОВАРЬ-ТЕЗАУРУС ЕВРАС, ТОМ 2, ОТ РЕАКЦИИ К СТИМУЛУ, ЧЕРКАСОВА Г.А., УФИМЦЕВА Н.В., 2014.

СТЕРНИН, И. А, РУДАКОВА, А. В., (2011). ПСИХОЛИНГВИСТИЧЕСКОЕ ЗНАЧЕНИЕ СЛОВА И ЕГО описАниЕ. Saarbrücken: Lambert Academic Publishing.

УФИМЦЕВА Н. В. (2013). СИСТЕМНО-ЦЕЛОСТНЫЙ ПРИНЦИП И АНАЛИЗ ЯЗЫКОВОЙ КАРТИНЫ МИРА. ЯЗЫК, СОЗНАНИЕ, КОММУНИКАЦИЯ. СС. 122-127.

УФИМЦЕВА, Н. В., ЧЕРКАСОВА, Г. А., КАРАУЛОВ, Ю. Н, ТАРАСОВ, Е. Ф. (2004). СЛАВЯНСКИЙ АССОЦИАТИВНЫЙ СЛОВАРЬ: РУССКИЙ, БЕЛОРУССКИЙ, БОЛГАРСКИЙ, УКРАИНСКИЙ. МОСКВА: ИНСТИТУТ ЯЗЫКОЗНАНИЯ РАН.

ШАПОШНИКОВА И. В. ПРАВОСЛАВНАЯ КУЛЬТУРА В НАУКЕ: АКСИОЛОГИЧЕСКАЯ эТНОПСИХОЛИНГВИСТИКА // воПРОСЫ ПСИХОЛИНГВИСТИКИ 2016 ВЫП. 2 № 28 стР. 302-323. ШАПОШНИКОВА И. В., РОМАНЕНКО А. А, РУССКИЙ РЕГИОНАЛЬНЫЙ АССОЦИАТИВНЫЙ СЛОВАРЬ (СИБИРЬ И ДАЛЬНИЙ ВОСТОК), Т.1 ОТ СТИМУЛА К РЕАКЦИИ, М. МОСКОВСКИЙ ИНСТИТУТ лингВИСТИКИ 2014

ШАПОШНИКОВА И. В., РОМАНЕНКО А. А, РУССКИЙ РЕГИОНАЛЬНЫЙ АССОЦИАТИВНЫЙ СЛОВАРЬ (СИБИРЬ И ДАЛЬНИЙ ВОСТОК), Т. Т. 2: ОТ РЕАКЦИИ К СТИМУЛУ / ОТВ. РЕД. Н.В. УФИМЦЕВА М.: московскиЙ инститУт лингвистики, 2015. 763с.

ШМЕЛЕВ А. Д. (2011). ДОПУСТИМО ЛИ ИЗУЧАТЬ РУсскИЙ ЯЗЫК? Toronto Slavic Quarterly. №31 


\section{NOTES}

1. Cette recherche est effectuée dans le cadre du projet commun RFFI-CNRS №19-512-15001 \19.

2. Dans le cadre des études dites "conceptuelles" il est usuel de les indiquer en majuscules.

3. Dans le dictionnaire de Kiss, les variantes orthographiques ont été comptabilisées à part.

\section{RÉSUMÉS}

Cet article présente l'état des lieux dans un domaine insuffisamment représenté en linguistique française, les dictionnaires d'associations évoquées par les mots. Seront présentés les principes sur lesquels ces dictionnaires sont construits, la méthodologie, les résultats et leur exploitation. Établis d'après les résultats d'enquêtes psycholinguistiques de fixation de la première réponse à un stimulus donné, ces dictionnaires se présentent sous deux formes - le dictionnaire direct, comportant les réactions classées par ordre de fréquence, et le dictionnaire inverse, dans lequel le mot vedette est la réaction, couplée aux stimuli qui l'ont provoquée. Différentes expériences menées au cours des 15 dernières années permettent de présenter la variation diatopique et diachronique des normes associatives du français.

The paper presents dictionaries of words associations, which are not frequently discussed in French linguistics. We introduce the base principles of word associations lexicology and the methodology used to build such dictionaries, then the results and their exploitation are explained. Those dictionaries are formed on psycholinguistic experiments where the first response to a given stimulus is fixed. There are two forms of dictionaries - the direct one, presenting the stimulus and all the obtained reactions in decreasing order of frequency, and the reverse one, where one can see every obtained reaction and all the stimuli that evoked it. Several experiments carried out over the past 15 years allow to present diatopic and diachronic variations in associative norms of French language.

\section{INDEX}

Mots-clés : associations évoquées par les mots, psycholinguistique, dictionnaire, français, diachronie

Keywords : word associations, psycholinguistics, dictionary, French language, diachrony

\section{AUTEUR \\ MICHĖLE DEBRENNE}

Université d'Etat de Novossibirsk, Institut de Sciences humaines 